\title{
TIME-DELAY CONTROL OF A MAGNETIC LEVITATED LINEAR POSITIONING SYSTEM
}

\author{
J.H.Tarn K.Y. Juang C.E. Lin \\ Institute of Aeronautics and Astronautics \\ National Cheng Kung University \\ Tainan, Taiwan
}

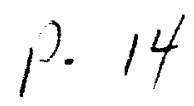

\begin{abstract}
In this paper, a high accuracy linear positioning system with a linear force actuator and magnetic levitation is proposed. By locating a permanently magnetized rod inside a current-carrying.solenoid, the axial force is achieved by the boundary effect of magnet poles and utilized to power the linear motion, while the force for levitation is governed by Ampere's Law supplied with the same solenoid. With the levitation in a radial direction, there is hardly any friction between the rod and the solenoid. The high speed motion can hence be achieved. Besides, the axial force acting on the rod is a smooth function of rod position, so the system can provide nanometer resolution linear positioning to the molecule size. Since the force-position relation is highly nonlinear, and the mathematical model is derived according to some assumptions, such as the equivalent solenoid of the permanently magnetized rod, so there exists unknown dynamics in practical application. Thus "robustness" is an important issue in controller design. Meanwhile the load effect reacts directly on the servo system without transmission elements, so the capability of "disturbance rejection" is also required. With the above consideration, a time-delay control scheme is chosen and applied. By comparing the input-output relation and the mathematical model, the time-delay controller calculates an estimation of unmodeled dynamics and disturbances and then composes the desired compensation into the system. Effectiveness of the linear positioning system and control scheme are illustrated with simulation results.
\end{abstract}

\section{INTRODUCTION}

Interest in research on large-gap magnetic suspension systems began in the early 1960's. The principal goal was the elimination of aerodynamic support interference in wind tunnel testing. To early 1970's the interest extended to small-gap ones. The first system developed was the Annular Momentum Control Device (AMCD) with applications to the stabilization and control of spacecraft [1]. This research was continued with the Annular Suspension and Pointing System (ASPS) which provides orientation, mechanical isolation, and fine pointing of space experiments[2][3]. For decades, Magnetic suspension technologies (MST) have demonstrated their capabilities in many fields, from industrial compressors, high-speed milling and grinding spindles, magnetically levitated trains, control wheel suspension for 
spacecraft to rocket propulsion turbomachinery. Important features of the magnetic suspensions and actuator systems are:

(1) Versatility of the Electromagnetic Forces

The physical force of a magnetic circuit to a high-permeable armature is called the Maxwell-force. Contrary to this commonly used force, the reaction force of a conductor carrying a current in a magnetic field is called the Lorentz-force. By successful integration of these physical effects, the constructed electromagnetic subsystem can be utilized as a rotary motor, linear actuator, radial bearing, thrust bearing, etc.

(2) Molecule-size Resolution

One problem of electric motors is the ripple of motion at low-speed operating regions due to the finite pole effect. The rotor always rests at the finite circumference positions which have the minimum magnetic flux (potential energy). Thus there are inherited limitations for resolution of control. The non-pole magnetic field provided by a coil, on the other hand, sets no resolution limitation. The resolution limit, in turn, is set by sensors, instrumentation and control strategies. Magnetic suspension systems provide a promising approach for achieving positioning with nanometer resolution.

In this paper, a linear positioning system with a linear force actuator and magnetic levitation is to be designed. By locating a permanently magnetized rod inside a current-carrying solenoid, the axial force is achieved by the boundary effect of magnet poles and utilized to power the linear motion, while the force for levitation is provided by the magnetic bearing and governedby the maximum linkage principle. With the levitation in a radial direction, there is no friction between the rod and solenoid. The demand of high-speed motion can hence be achieved. Under the proposed arrangement, the axial force acting on the rod is a smooth function of rod position, so the system can provide nanometer-resolution linear positioning to the molecule size. It is known that an effective control system is the key condition for successful implementation of high-performance magnetic levitated positioning systems. Major issues for design of such control systems are:

(1) Nonlinearity

By assuming that the complete energy of the magnetic field is concentrated within the air gap. The basic mathematical models of active magnetic bearings are obtained from Maxwell's laws. The input-output relations are highly nonlinear despite the variables defined.

(2) Unmodelled Dynamics

Secondary effects such as copper resistance, stray fields and saturation are neglected.

(3) Disturbance Rejection

Because the load effect reacts directly on the servo system without the transmission elements, the capability of "disturbance rejection" is also required.

With the above considerations, a time-delay control scheme with the properties of "robustness" and "disturbance rejection" is utilized[4][5]. By comparing the input-output relation and the mathematical model, the time-delay controller calculates an estimation of unmodeled dynamics and disturbances and then composes the desired compensation into the system. Effectiveness of the linear positioning and control systems is illustrated by numerical simulation results. 


\title{
SYSTEM DYNAMICS
}

\author{
System Configuration
}

The configuration of the proposed magnetic levitated linear positioning system is shown in Figure 1. To achieve the function of levitation, the current in the solenoid must be kept in the direction that can maintain the stability of radial motion. Under such condition, the magnetic force in the axial direction tends to push the rod away from the center of the solenoid. Hence a spring is required to supply the force in the opposite direction. Also, the spring must be precompressed to avoid an uncontrollable equilibrium point. The additional magnetic bearing system is used to keep the moving part balanced in the axial direction. With a biased current fed to the solenoid, the magnetic force $(F=I \times B)$ in the radial direction is utilized to suspend the moving part, while, with the controlled current, the axial motion is governed by the force caused by the non-uniform magnetic field in the boundary.

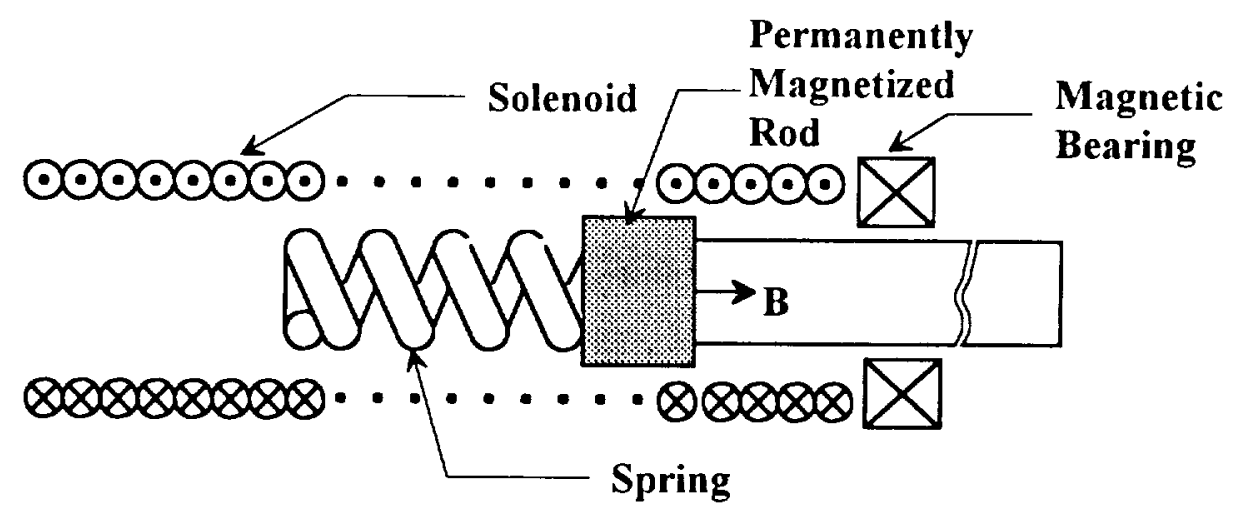

Figure 1 The configuration of magnetic levitated linear positioning system

\author{
Dynamics in Axial Motion
}

To derive the dynamics, a solenoid is employed to produce the equivalent magnetic field $\mathbf{B}$ of the permanently magnetized rod and thus simplify the calculation of the force-position relationship. With such an arrangement, the solenoid-rod configuration can be approximated as depicted in Figure 2 . The force acting on the inner coil due to the current in the outer coil can then be found with Ampere's Law. 


\section{$\odot 0000000 \ldots \ldots . . . .00000$ \\ $\$ 80 \cdot \cdot \otimes$ \\ $\odot \odot \odot \cdot \odot$ \\ $\$ 8080800 . \ldots . . . \otimes 8880$}

Figure 2 Equivalent configuration

Consider two current-carrying elements as shown in Figure 3. The force between the two current-carrying elements is[6]

$$
d \mathbf{F}=\frac{\mu_{0} I_{2} I_{1}}{4 \pi r^{2}} \hat{\mathbf{a}}_{1} \times\left(\hat{\mathbf{a}}_{2} \times \hat{\mathbf{r}}\right) d l_{2} d l_{1}
$$

where $\quad d \mathbf{F}=$ force on element 1 due to current in element $2, \mathrm{~N}$

$\mu_{0}=$ permeability of air, $\mathrm{Hm}^{-1}$

$d l_{1}, d l_{2}=$ lengths of current-carriyng elements 1 and 2 , respectively, $\mathrm{m}$

$I_{1}, I_{2}=$ currents in elements 1 and 2 , respectively, A

$r=$ distance between elements, $\mathrm{m}$

$\hat{\mathbf{a}}_{1}=$ unit vector in direction of current in element 1 , dimensionless

$\hat{\mathbf{a}}_{2}=$ unit vector in direction of current in element 2 , dimensionless

$\hat{\mathbf{r}}=$ unit vector in radial direction( from element 2 to 1 ), dimensionless

Applying the force-position relation to the equivalent model, it can be found that the force acting on the inner coil with radius $r_{2}$ at position $x_{2}$ due to the current-carrying outer coil with radius $r_{1}$ at position $x_{1}$ is given by the following equation:

$$
\begin{aligned}
d \mathbf{F} & =\frac{\mu_{0} I_{1} I_{2} r_{1} r_{2} d \theta_{1} d \theta_{2}}{4 \pi\left[\left(x_{1}-x_{2}\right)^{2}+\left(r_{1} \sin \theta_{1}-r_{2} \sin \theta_{2}\right)^{2}+\left(r_{1} \cos \theta_{1}-r_{2} \cos \theta_{2}\right)^{2}\right]^{3 / 2}} \cdot\left[\cos \theta_{2} \vec{j}-\sin \theta_{2} \vec{k}\right] \\
& \times\left\{\left[\cos \theta_{1} \vec{j}-\sin \theta_{1} \vec{k}\right] \times\left[\left(x_{2}-x_{1}\right) \vec{i}+\left(r_{2} \sin \theta_{2}-r_{1} \sin \theta_{1}\right) \vec{j}+\left(r_{2} \cos \theta_{2}-r_{1} \cos \theta_{1}\right) \vec{k}\right]\right\}
\end{aligned}
$$

Hence the force in the axial direction can be expressed as

$$
d \mathbf{F}_{x}=\frac{-\mu_{0} I_{1} I_{2} r_{1} r_{2}\left(x_{2}-x_{1}\right)\left(\cos \theta_{1} \cos \theta_{2}+\sin \theta_{1} \sin \theta_{2}\right)}{4 \pi\left[\left(x_{1}-x_{2}\right)^{2}+\left(r_{1} \sin \theta_{1}-r_{2} \sin \theta_{2}\right)^{2}+\left(r_{1} \cos \theta_{1}-r_{2} \cos \theta_{2}\right)^{2}\right]^{3 / 2}} d \theta_{1} d \theta_{2}
$$

with the numerical calculation, the force-position relation of the rod, with 5000 Gauss of flux density at one end on the axis shown in Figure 4, where $r_{1}$ is $1.1 \mathrm{~cm}, r_{2}$ is $1.0 \mathrm{~cm}$, the length of the rod is $1.0 \mathrm{~cm}$, and the length of the solenoid is $10 \mathrm{~cm}$. The result indicates that the solenoid-rod configuration operates as a current-controlled stiffness nonlinear spring, so the model of axial motion can be simplified and shown in Figure 5, where $K_{1}$ is the preload spring, and $K_{2}$ is the equivalent model of the solenoid-rod configuration. 


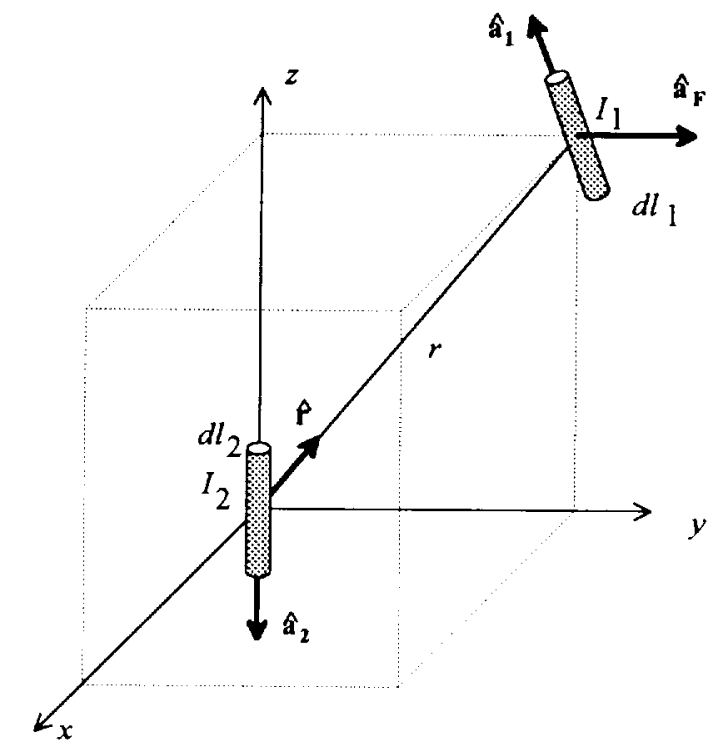

Figure 3 Geometry of short current-carrying elements for finding force between them.

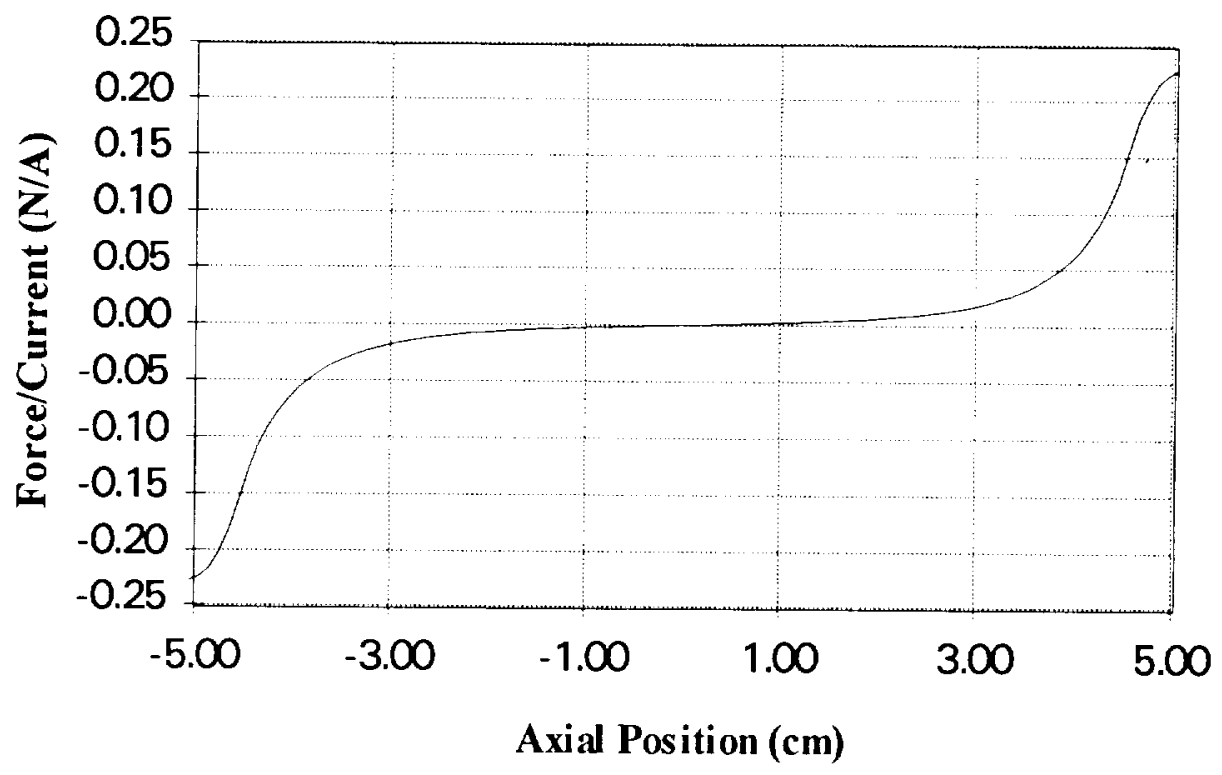

Figure 4 Force-position relation in axial motion.

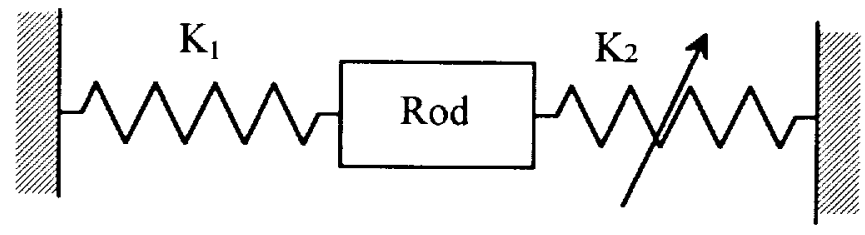

Figure 5 Simplified model of axial motion. 
The dynamic equation in axial motion can then be modeled as

$$
\begin{gathered}
\dot{x}_{1}=x_{2} \\
\dot{x}_{2}=-\frac{1}{m}\left[K_{1} \cdot\left(x_{1}+x_{p}\right)+K_{2}\left(x_{1}\right) \cdot\left(i+i_{b}\right)\right]
\end{gathered}
$$

where

$x_{1}, x_{2}=$ position and velocity of the rod respectively, $\mathrm{cm}, \mathrm{cm} / \mathrm{sec}$

$K_{1}=$ stiffness of the spring, $\mathrm{N} / \mathrm{cm}$

$K_{2}(x)=$ current controlled stiffness of solenoid-rod configuration, N/A

$x_{p}=$ pre-compressed length of the spring, $\mathrm{cm}$

$i_{b}=$ biased current for levitation, A

$i=$ controlled current, A

$m=$ mass of the magnetic rod, $\mathrm{kg}$

\section{Dynamics of Radial Motion}

To verify the function of levitation of the proposed mechanism, the dynamics of radial motion is taken into consideration. Imagine a small deviation of the rod position in radial direction $y_{2}$, then the radial force can be modeled as

$$
d \mathbf{F}_{y}=\frac{\mu_{0} I_{1} I_{2} r_{1} r_{2}\left[r_{2} \sin \theta_{2}\left(\cos \theta_{1} \cos \theta_{2}+\sin \theta_{1} \sin \theta_{2}\right)+\left(y_{2} \sin \theta_{1}-r_{1}\right) \sin \theta_{2}\right]}{4 \pi\left[\left(x_{1}-x_{2}\right)^{2}+\left(y_{2}+r_{1} \sin \theta_{1}-r_{2} \sin \theta_{2}\right)^{2}+\left(r_{1} \cos \theta_{1}-r_{2} \cos \theta_{2}\right)^{2}\right]^{3 / 2}} d \theta_{1} d \theta_{2},
$$

From the calculation, the result is shown in Figure 6. It is clear that, with positive current, the force tends to force the rod staying in the central axial position. Any deviation in radial direction will cause a force in the opposite direction to push it back. Hence the function of levitating is achieved. In this paper, the motion in the radial direction is not controlled.

Since the system is passive and motion in the radial direction is confined, the open loop in the radial direction poses no stability problem. 


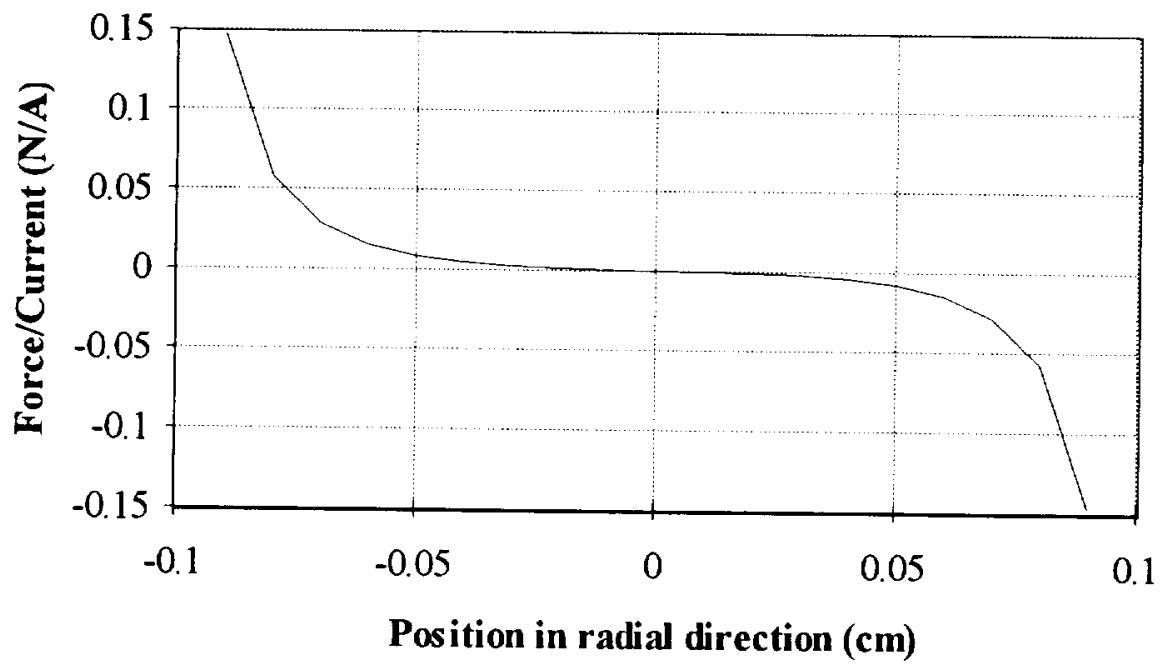

Figure 6 Axial force-position relation.

\title{
CONTROLLER DESIGN
}

\author{
Time Delay Controller
}

Since the magnetic levitated positioning system is subjected to the load effect directly, the controller must be good at disturbance rejection. Besides, the force-position relation is highly nonlinear. Hence the Time-Delay Controller (TDC) design method proposed by Youcef-Toumi [3] is applied to control the rod position.

Consider the axial motion to be controlled as

$$
\dot{\mathbf{x}}=\mathbf{f}(\mathbf{x}, t)+\mathbf{h}(\mathbf{x}, t)+\mathbf{B}(\mathbf{x}, t) \mathbf{u}+\mathbf{d}(t)
$$

where

$\mathbf{x}=$ an $n \times 1$ plant state vector,

$\mathbf{f}(\mathbf{x}, t)=$ an $n \times 1$ nonlinear vector represents the known dynamics,

$\mathbf{h}(\mathbf{x}, t)=$ an $n \times 1$ nonlinear vector represents the unknown dynamics,

$\mathbf{B}(\mathbf{x}, t)=$ an $n \times r$ control distribution matrix with rank $r$,

$\mathrm{d}(t)=$ an $n \times 1$ unknown disturbance function.

Define the linear time-invariant reference model as

$$
\dot{\mathbf{x}}_{\mathrm{m}}=\mathbf{A}_{\mathrm{m}} \mathbf{x}_{\mathrm{m}}+\mathbf{B}_{\mathrm{m}} \mathbf{r}
$$


and the error between the model and the plant as

$$
\mathbf{e}=\mathbf{x}_{\mathbf{m}}-\mathbf{x}
$$

The objective of the control scheme is to make the error behave as the desired dynamics

$$
\dot{\mathbf{e}}=\mathbf{A}_{\mathbf{e}} \mathbf{e}=\left(\mathbf{A}_{\mathbf{m}}+\mathbf{K}\right) \mathbf{e}
$$

Combined with the plant dynamics and reference model dynamics, the error dynamics can be formulated as

$$
\dot{\mathbf{e}}=\mathbf{A}_{\mathbf{e}} \mathbf{e}+\left[-\mathbf{f}-\mathbf{h}-\mathbf{d}+\mathbf{A}_{\mathbf{m}} \mathbf{x}+\mathbf{B}_{\mathbf{m} \mathbf{r}} \mathbf{r}-\mathbf{B} \mathbf{u}-\mathbf{K e}\right]
$$

To satisfy the desired error dynamics, the control input u must be functioned to make

$$
\left[-\mathbf{f}-\mathbf{h}-\mathbf{d}+\mathbf{A}_{\mathbf{m}} \mathbf{x}+\mathbf{B}_{\mathbf{m}} \mathbf{r}-\mathbf{B u}-\mathbf{K e}\right]=\mathbf{0}
$$

Observe the above equation; only $\mathbf{h}$ and $\mathbf{d}$ are unknown. They can be estimated from the measured information at time $t-L$, with

$$
\hat{\mathbf{h}}(\mathbf{x}, t)+\mathbf{d}(t) \cong \hat{\mathbf{h}}(\mathbf{x}, t-L)+\mathbf{d}(t-L) \cong \dot{\mathbf{x}}(t-L)-\mathbf{f}(\mathbf{x}, t-L)-\mathbf{B}(\mathbf{x}, t-L) \mathbf{u}(t-L)
$$

Then the TDC control law is

$$
\mathbf{u}(t)=\mathbf{B}^{+}(t)\left[\mathbf{f}(\mathbf{x}, t-L)-\dot{\mathbf{x}}(t-L)+\mathbf{B}(\mathbf{x}, t-L) \mathbf{u}(t-L)-\mathbf{f}(t)+\mathbf{A}_{\mathbf{m}} \mathbf{x}(t)+\mathbf{B}_{\mathbf{m}} \mathbf{r}(t)-\mathbf{K e}(t)\right]
$$

Rewrite the dynamics equation of the magnetic levitated linear positioning system as

$$
\begin{gathered}
\dot{x}_{1}=x_{2} \\
\dot{x}_{2}=-\frac{1}{m}\left\{\left[K_{1} \cdot\left(x_{1}+x_{p}\right)\right]+\left[K_{2}\left(x_{1}\right) \cdot\left(i+i_{b}\right)+m \hat{b} i\right]\right\}+\hat{b} i
\end{gathered}
$$

and let

$$
\mathbf{f}(\mathbf{x})=\left[\begin{array}{c}
x_{2} \\
-K_{1} \cdot\left(x_{1}+x_{p}\right) / m
\end{array}\right], \mathbf{h}(\mathbf{x})=\left[\begin{array}{c}
0 \\
-K_{2}\left(x_{1}\right) \cdot\left(i+i_{b}\right) / m-\hat{b} i
\end{array}\right] \text { and } \mathbf{B}=\left[\begin{array}{l}
0 \\
\hat{b}
\end{array}\right]
$$

then by choosing the reference model as

$$
\left[\begin{array}{l}
\dot{x}_{1} \\
\dot{x}_{2}
\end{array}\right]=\left[\begin{array}{cc}
0 & 1 \\
-100 & -20
\end{array}\right]\left[\begin{array}{l}
x_{1} \\
x_{2}
\end{array}\right]+\left[\begin{array}{c}
0 \\
100
\end{array}\right] i
$$

and $\mathbf{K}=0$, then the control force is 


$$
i(t)=\hat{b}^{-1}\left\{K_{1}\left[x_{1}(t-L)-x_{1}(t)\right] / m-\dot{x}_{2}(t-L)+\hat{b}(t-L) i(t-L)-100 x_{1}(t)-20 x_{2}(t)+100 r(t)\right\}
$$

The simulation results are shown in Figure 7 and Figure 8 . In the simulation, the control force is limited with a saturation bound $[-3.5,10]$ and $\hat{b}$ is chosen to be a constant 10 . Under the case in Figure 7 , the linear positioning system is subjected to a step disturbance in the axial direction at time $t=0.5 \mathrm{sec}$, whose amplitude is -0.1 . For the case in Figure 8, the system is utilized to move a load with mass equals to $5 \mathrm{~m}$, the mass of the moving part.
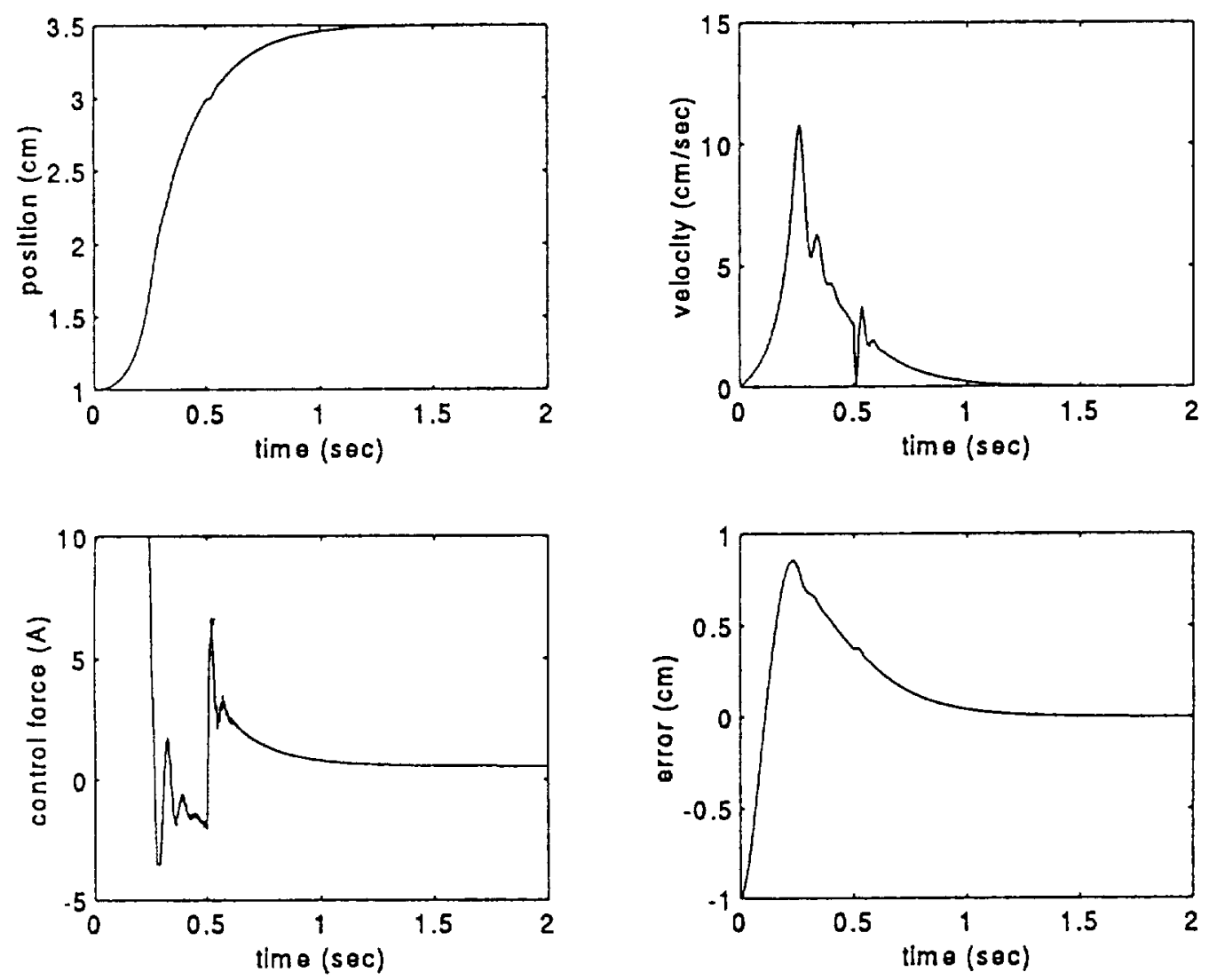

Figure 7 Simulation result with Time Delay Controller. 

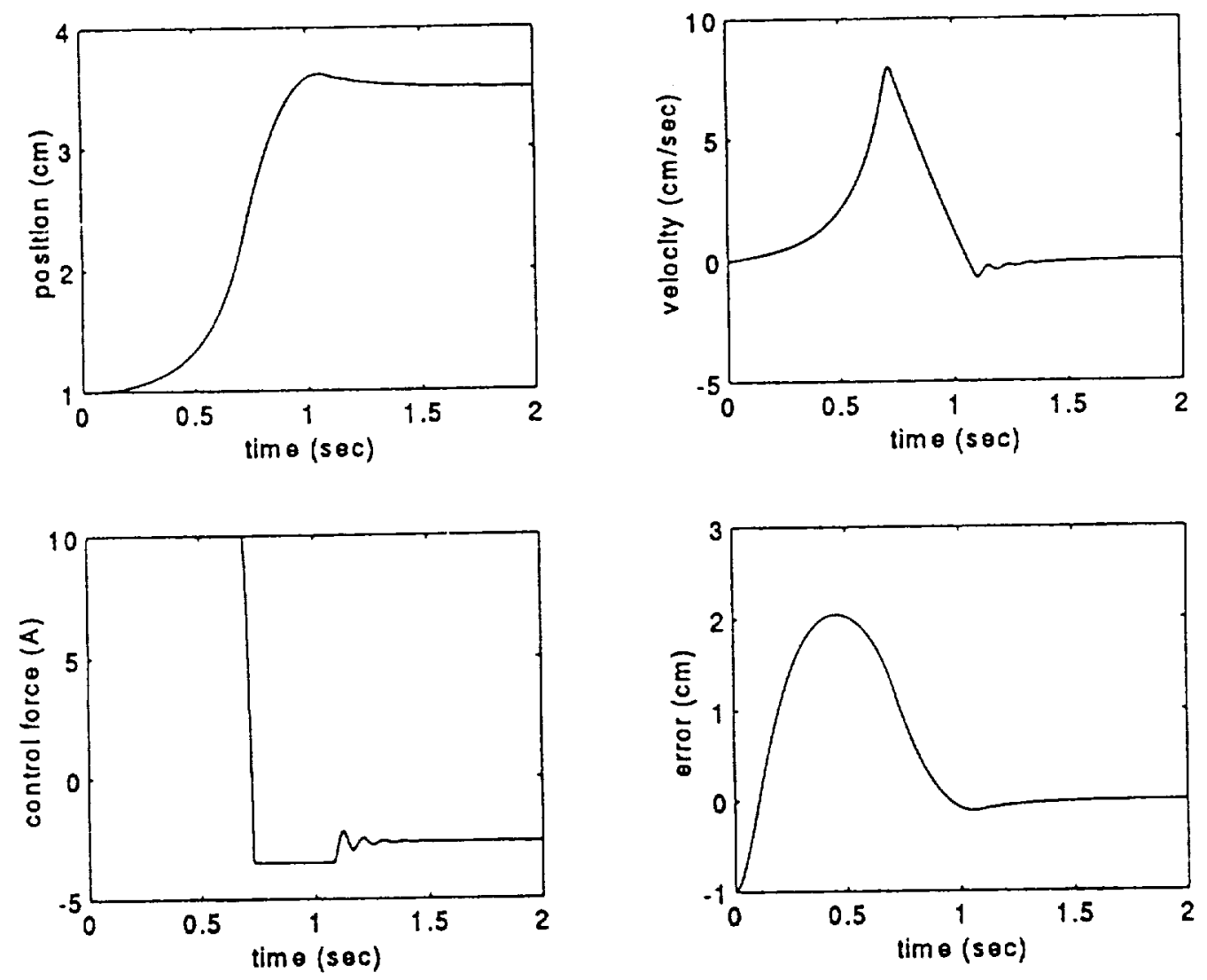

Figure 8 Simulation result of TDC

Time Delay Controller with Mean Value Theorem

The method described in the previous section requires the measurement of $\dot{x}_{2}$. It means that the designer must take care of either the noise induced by numerical derivativeness or the setting up of the accelerometer. An alternative scheme is introduced by Youcef-Toumi[4] with the application of the mean-value theorem as follows:

Define the unmodeled dynamics $\Psi=\mathbf{h}+\mathbf{d}$; the error dynamics can be written as

$$
\dot{e}=A_{e} e+\left[-\mathbf{f}-\Psi+A_{m} \mathbf{x}+B_{m} \mathbf{r}-B u-K e\right]
$$

Integrate the equation at both sides to get

$$
\mathbf{e}(t)=\Phi\left(t, t_{0}\right) \mathbf{e}\left(t_{0}\right)+\int_{t_{0}}^{t} \Phi(t, \tau)\left[\mathbf{A}_{\mathrm{m}} \mathbf{x}(\tau)+B_{\mathrm{m}} \mathbf{r}(\tau)-\mathbf{f}(\tau)-\Psi(\tau)-B \mathbf{B}(\tau)-\operatorname{Ke}(\tau)\right] d \tau \quad \forall t \geq t_{0}
$$


where

$$
\Phi(t, \tau)=e^{\mathbf{A}_{\mathrm{e}}(l-\tau)}
$$

Then

$$
\int_{t_{0}}^{t} \Phi(t, \tau) \Psi(\tau) d \tau=\Phi\left(t, t_{0}\right) \mathbf{e}\left(t_{0}\right)-\mathbf{e}(t)+\int_{t_{0}}^{t} \Phi(t, \tau)\left[\mathbf{A}_{\mathbf{m}} \mathbf{x}(\tau)+\mathbf{B}_{\mathbf{m}} \mathbf{r}(\tau)-\mathbf{f}(\tau)-\mathbf{B u}(\tau)-\mathbf{K e}(\tau)\right] d \tau
$$

Utilizing the mean-value theorem approximation, the above equation can be approximated by

$$
\left[\int_{t_{0}}^{t} \Phi(t, \tau) d \tau\right] \hat{\mathbf{Y}}(t)=\Phi\left(t, t_{0}\right) \mathbf{e}\left(t_{0}\right)-\mathbf{e}(t)+\int_{t_{0}}^{t} \Phi(t, \tau)\left[\mathbf{A}_{\mathbf{m}} \mathbf{x}(\tau)+\mathbf{B}_{\mathbf{m}} \mathbf{r}(\tau)-\mathbf{f}(\tau)-\mathbf{B u}(\tau)-\mathbf{K e}(\tau)\right] d \tau
$$

Thus the control force can be found as

$$
\mathbf{u}=\mathbf{B}^{+}\left[\mathbf{A}_{\mathbf{m}} \mathbf{x}+\mathbf{B}_{\mathbf{m}} \mathbf{r}-\mathbf{f}-\hat{\Psi}-\mathbf{K e}\right]
$$

With the same choice of reference model in the previous section, the simulation result with step disturbance in axial force is given in Figure 9. Figure 10 demonstrates the result of load effect.
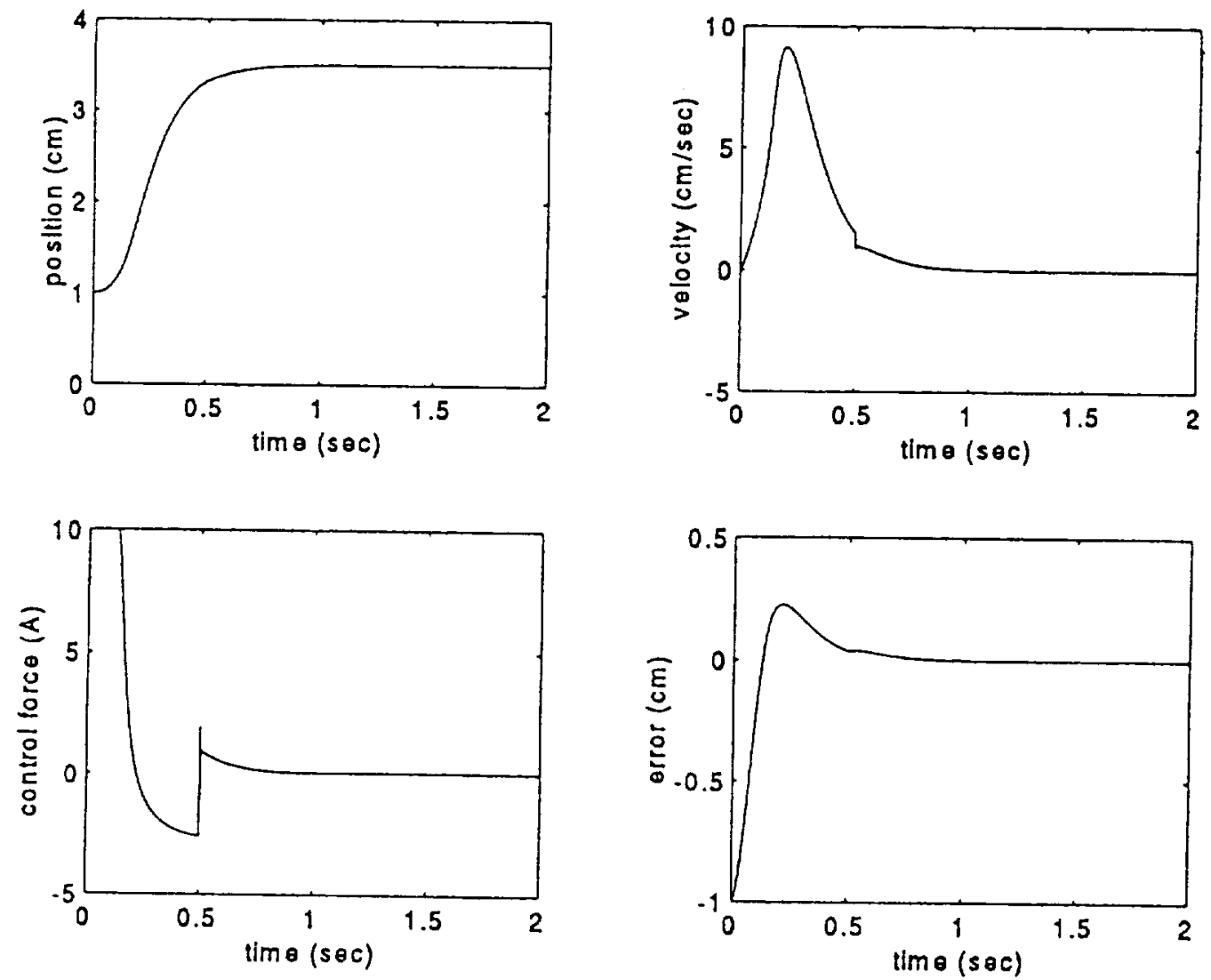

Figure 9 Simulation result with modified Time Delay Controller (I) 

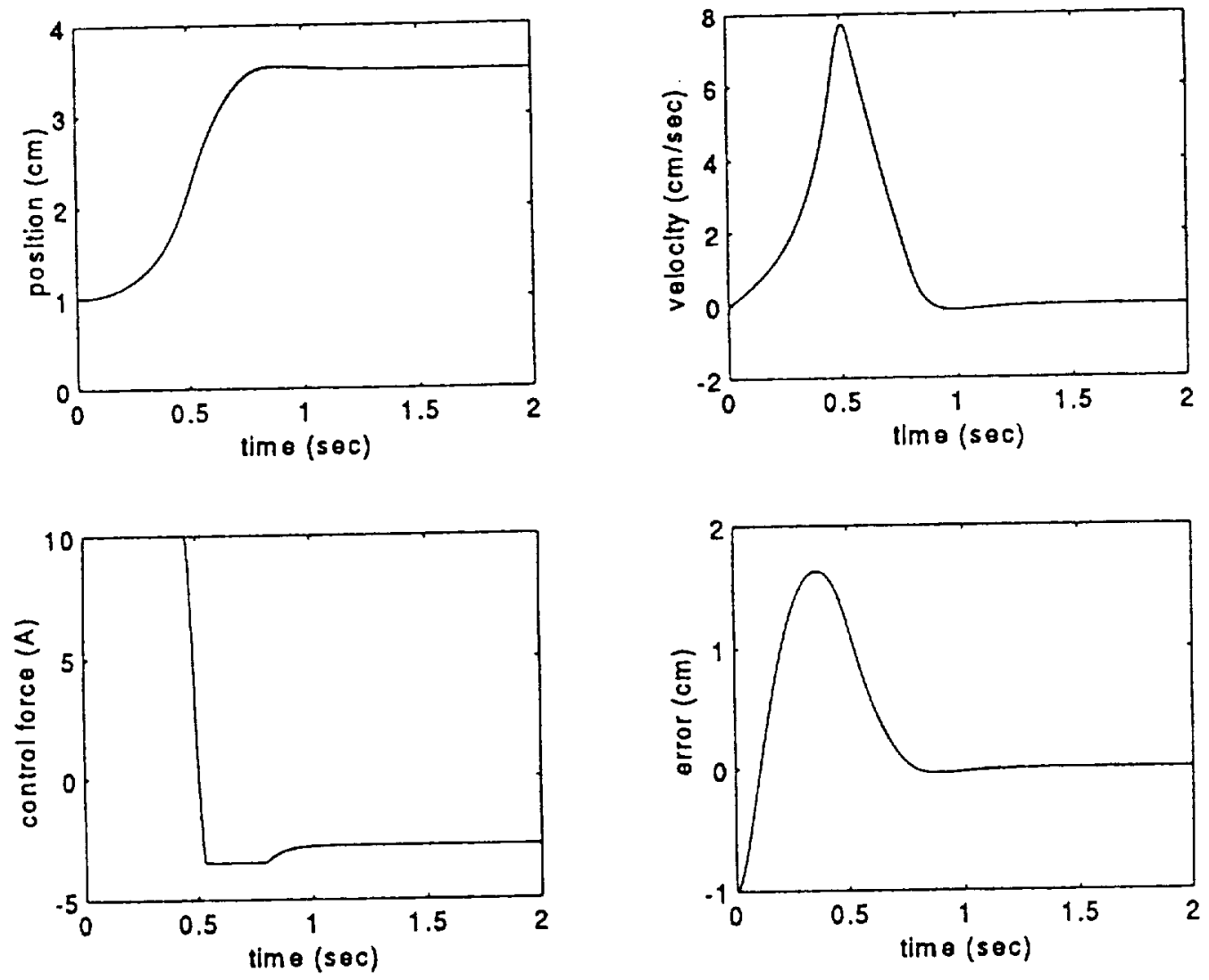

Figure 10 Simulation result with modified Time Delay Controller (II)

\section{Discussions}

From the simulation results, the property of "disturbance rejection" of Time Delay Controllers is demonstrated. Due to the saturation constraint in the control force, the controller can perform well with limited external load. With the mean-value approximation, the performance is improved. But it requires a higher sampling rate to gather more information in the delay time.

During the design stage, the choice of parameter $\hat{b}$ plays an important role. In general, if we know more about the nonlinear dynamics of the system to be controlled, we can have a better choice of $\hat{b}$. Under the situation that no information is given for the unknown dynamics, we usually choose $\hat{b}$ as a constant value. From simulation results shown in Figure 11, we conclude that the larger $\hat{b}$ provides more guaranteeon the "disturbance rejection", but has poorer performance. But, the larger $\hat{b}$ drives the control force to the saturation bound easily and consequently the system fails to work. 


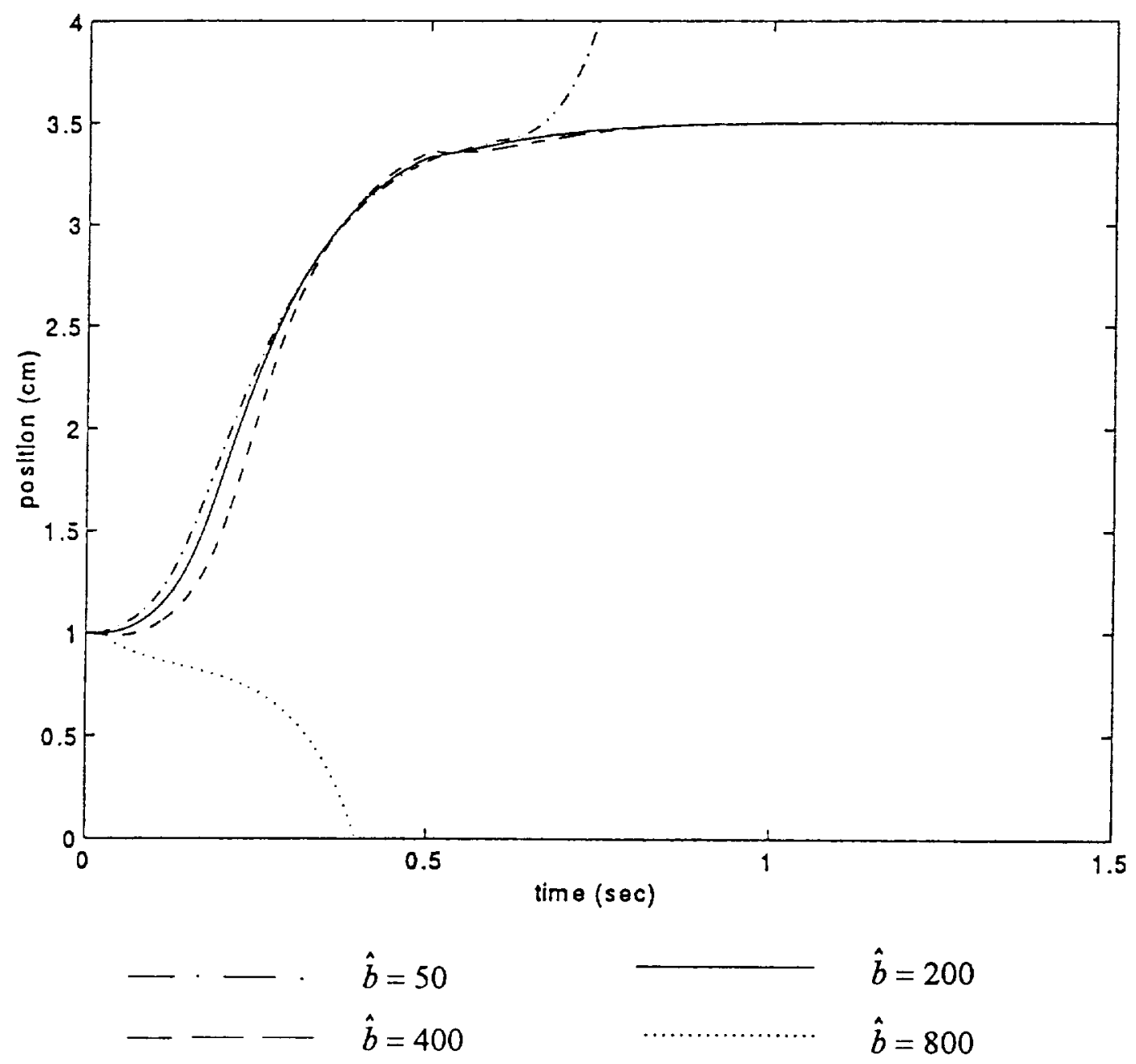

Figure 11 Response under different choices of $\hat{b}$.

\section{CONCLUSION}

Applying the equivalent magnetic flux density between the solenoid and permanently magnetized rod, a method of modeling the force-position relation in the solenoid-rod configuration is proposed. Based on the model, a "current controlled stiffness" spring serves as a low friction linear positioning system. Two kinds of Time Delay Control schemes are applied to control the system. The Time Delay Controller is a fast adaptive control scheme without identification of the dynamics of the plant to be controlled, so it can be easily implemented. From the simulation results, the property of disturbance rejection is demonstrated. 


\section{REFERENCES}

[1] Anderson, Willard W. and Groom, Nelson J., "The Annular Momentum Control Device (AMCD) and Potential Applications," NASA TN D-7866, 1975.

[2] Anderson, Willard W. and Joshi, Suresh M., "The Annular Suspension and Pointing (ASP) System for Space Experiments and Predicted Pointing Accuracies," NASA TR R-448, 1975.

[3] Anderson, Willard W; Groom, Nelson J. and Woolley, Charles T.,"The Annular Suspension and Pointing System," Journal of Guidance and Control, Vol. 2, No. 5, 1979, pp. 367-373.

[4] Youcef-Toumi, K. and Wu, S.-T., 1992, "Input/Output Linearization Using Time Delay Control," ASME Journal of Dynamic Systems, Measurement, and Control, March issue, 1992.

[5] Youcef-Toumi, K. and Reddy, S., "Analysis of Linear Time Invarient Systems with Time Delay," ASME Journal of Dynamic Systems, Measurement, and Control, December issue, 1992.

[6] Kraus, J. D."Electromagnetic," 3rd Edition, McGraw-Hill.

[7] Youcef-Toumi, K. and Ito, O., "A Time Delay Controller for Systems With Unknown Dynamics," ASME Journal of Dynamic Systems, Measurement, and Control, Vol. 112, Mar 1990, pp.133-142.

[8] Youcef-Toumi, K., "Fast Adaptive Control Algorithms In Precision Motion Control Systems," Proceedings of 1993 National Symposium on Automatic Control, pp. 59-149. 